Research Paper

\title{
No Resistance to Penicillin, Cefuroxime, Cefotaxime, or Vancomycin in Pneumococcal Pneumonia
}

\author{
Josef Yayan ${ }^{1 凶}$, Beniam Ghebremedhin ${ }^{2}$, Kurt Rasche ${ }^{1}$ \\ 1. Witten/Herdecke University, Witten, Department of Internal Medicine, Division of Pulmonary, Allergy, and Sleep Medicine, HELIOS Clinic \\ Wuppertal, Germany \\ 2. Witten/Herdecke University, Witten, Institute of Medical Laboratory Diagnostics, Center for Clinical and Translational Research, HELIOS Clinic \\ Wuppertal, Germany
}

$\triangle$ Corresponding author: Josef Yayan, MD, Department of Internal Medicine, Division of Pulmonary, Allergy, and Sleep Medicine, HELIOS Clinic Wuppertal, Witten/Herdecke University, Heusnerstr. 40, 42283 Wuppertal, Germany. Tel: +49 0202896 3973; Fax: +49 0202896 3941; Email: josef.yayan@hotmail.com

(C) 2015 Ivyspring International Publisher. Reproduction is permitted for personal, noncommercial use, provided that the article is in whole, unmodified, and properly cited. See http://ivyspring.com/terms for terms and conditions.

Received: 2015.07.09; Accepted: 2015.10.26; Published: 2015.11.22

\begin{abstract}
Objectives: Group B Streptococcus is a primary source of pneumonia, which is a leading cause of death worldwide. During the last few decades, there has been news of growing antibiotic resistance in group B streptococci to penicillin and different antibiotic agents. This clinical study retrospectively analyzes antimicrobial resistance in inpatients who were diagnosed with group $B$ streptococcal pneumonia.

Methods: All of the required information from inpatients who were identified to have group $B$ streptococcal pneumonia was sourced from the database at the Department of Internal Medicine of HELIOS Clinic Wuppertal, Witten/Herdecke University, in Germany, from 2004-2014. Antimicrobial susceptibility testing was performed for the different antimicrobial agents that were regularly administered to these inpatients.

Results: Sixty-six inpatients with a mean age of $63.3 \pm 16.1$ years $(45$ males $[68.2 \%, 95 \% \mathrm{Cl}$ $60.0 \%-79.4 \%$ ] and 21 females [31.8\%, $95 \% \mathrm{Cl} 20.6 \%-43.0 \%]$ ) were detected to have group $\mathrm{B}$ streptococcal pneumonia within the study period from January 1, 2004, to August 12, 2014. Group B Streptococcus had a high resistance rate to gentamicin (12.1\%), erythromycin (12.1\%), clindamycin (9.1\%), and co-trimoxazole (3.0\%), but it was not resistant to penicillin, cefuroxime, cefotaxime, or vancomycin $(P<0.0001)$.

Conclusion: No resistance to penicillin, cefuroxime, cefotaxime, or vancomycin was detected among inpatients with pneumonia caused by group $B$ streptococci.
\end{abstract}

Key words: Antibiotic, Pneumonia, Resistance, Sensitivity, Group B Streptococcus

\section{Introduction}

Group B Streptococcus (GBS) is still a main cause of both community- and nosocomial-acquired pneumonia, and the associated mortality rates are increasing significantly around the world [1]. At the beginning of the antibiotic era in the mid-1970s, GBS was susceptible to all classes of antibiotics, such as penicillin, cephalosporins, and erythromycin [2]. Over the following decades, GBS became resistant to a variety of antibiotics, evolving from a considerable medical curiosity to a worldwide health problem. The treat- ment outcomes in cases of pneumonia due to antibiotic-resistant GBS have not been studied enough to understand the clinical significance [3].

This study was performed to discover which antibiotics were ineffective in the management of group B streptococcal pneumonia during the past 10 years. All necessary data for this study were sourced from the hospital records of inpatients who were treated because of community- or nosocomial-acquired group B streptococcal pneumonia, according to the Interna- 
tional Statistical Classification of Diseases (ICD J15.3), at the Department of Internal Medicine of the HELIOS Clinic, Witten/Herdecke University, Wuppertal, Germany $[4,5]$.

This comprehensive investigation was performed to detect antibiotic resistance over a 10-year period according to susceptibility testing in the tracheal or bronchial secretions and blood cultures of patients with group B streptococcal pneumonia. The number of antibiotics used and the failure of antimicrobial treatments were monitored in the study population during the study period. The rapid identification of an effective antibiotic for GBS would shorten both the duration of the disease and the length of the hospital stay, and patient mortality should be reduced, as well.

\section{Material and Methods}

\section{Patients}

This qualitative, observational control study retrospectively examined antibiotic resistance in patients who were identified to have group B streptococcal pneumonia. All of the required information was retrieved from the hospital database of the HELIOS Clinic, Witten/Herdecke University, Wuppertal, Germany, from January 1, 2004, to August 12, 2014. All patients with pneumonia caused by GBS and who were over 18 years of age were considered for this clinical study.

\section{Definition of Pneumonia}

Acute infection of lung tissue due to social contact is referred to as community-acquired pneumonia, whereas nosocomial-acquired pneumonia is developed during a stay in the hospital [5]. The final diagnosis of either community- or nosocomial-acquired pneumonia is made using $\mathrm{X}$-ray investigations and sputum analysis [6,7]. The specific criteria that were used for the clinical diagnosis of pneumonia in the present study were that the patient was hospitalized, exhibited the presence of new areas of infiltration on chest X-ray, and had new clinical symptoms, including a minimum of two of the following: difficulty breathing, fever over $38^{\circ} \mathrm{C}$, sputum production, or cough.

\section{Tested Antibiotics}

The susceptibility of GBS to the following antimicrobial agents was examined with the PHOENIXTM automated system (Becton Dickinson, Heidelberg, Germany): penicillin, ampicillin, piperacillin, ampicillin-sulbactam, piperacillin-tazobactam, cefuroxime, cefotaxime, ciprofloxacin, levofloxacin, erythromycin, co-trimoxazole, clindamycin, gentamicin, and vancomycin.
The number of times that each antimicrobial agent was administered for the management of group B streptococcal pneumonia during hospitalization was calculated. The antibiotic that was the most commonly used for treatment and the most often tested for antibiotic susceptibility was compared with the other antimicrobial agents. The antibiotic with the lowest resistance rate was also compared with the other antibiotics tested in the antibiograms.

For GBS, inhibition zone diameter breakpoints were used in keeping with the Clinical and Laboratory Standards Institute (CLSI) 2004-2011 laboratory agreement guidelines [8]. In 2011, the Europe-wide standards for susceptibility testing (EUCAST) were adopted in place of the CLSI's for this study because the EUCAST sets standards for almost all of the pathogens on which our tests are based [9].

\section{Identification and Antimicrobial Susceptibility Testing}

GBS was identified based on growth on Columbia blood agar (Becton Dickinson, Heidelberg, Germany) after $18-48$ hours at $37^{\circ} \mathrm{C}$ in $5 \% \mathrm{CO}_{2}$, as well as with the use of MALDI-TOF-MS (Bruker, Bremen, Germany). Software suitable for the interpretation of susceptibility testing results using the EUCAST breakpoints for 2011-2014 was used in the antimicrobial susceptibility testing [9].

The disc diffusion method established by Kirby-Bauer was used for the susceptibility testing to detect antibiotic resistance [10]. In cases of discrepancies or insufficient readings, the calculation of the minimum inhibitory concentration (MIC) was carried out with the E-test for particular antimicrobials, and the results were determined in accordance with the criteria of the EUCAST [9]. Intermediate isolates were grouped along with resistant isolates.

\section{Microbiology}

The diagnostic procedures used were tracheal washes during bronchoscopy, tracheal secretions, throat smears, and expectoration. For bronchoscopy, approximately $20 \mathrm{ml}$ of isotonic saline solution was infused into the bronchi after the patient was given local anesthesia, and washings were collected using the flexible fiber-optic bronchoscope into three separate, antiseptic, 40-ml specimen containers (Argyle ${ }^{\mathrm{TM}}$, Covidien, Neustadt an der Donau, Germany). Throat smears were taken using commercially available cotton sticks (MEUS Srl Ltd., Piove di Sacco, Italy), turning the cotton stick while pressing lightly on the pharynx of patients suspected to have pneumonia. Expectorates were collected in 30-ml sterilized reservoirs for expectoration (Salivette ${ }^{\circledR}$, SARSTEDT, Nümbrecht, Germany). 
Thus obtained, the bronchial and tracheal secretions and the sputum were examined after Gram staining under light microscopy at 80-1,000-times enlargement with a minimum of five fields of vision, in accordance with the criteria created by Bartlett [11].

\section{Blood Cultures}

To detect GBS from the bloodstream, approximately $20 \mathrm{ml}$ of blood was collected by puncturing a vein using a blood-collection needle, and then vaccinated into two special blood-culture media, BD

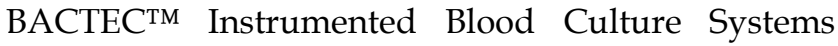
(Becton Dickson, Dickinson and Company, Heidelberg, Germany).

The duration of the hospital stay was evaluated in all inpatients diagnosed with group B streptococcal pneumonia. Additionally, the number of actual deaths during hospital stays was analyzed in the study population, and the survival probabilities were calculated according to the Kaplan-Meier method.

\section{Ethics Statement}

The ethics committee of Witten/Herdecke University examined this investigation as well as all investigational procedures. After approval of this trial, the committee waived the requirement for obtaining written consent due to the retrospective study design.

\section{Statistical Analysis}

Categorical numbers are stated as percentages, and numeric variables are given as means and standard deviations. The statistical computations were executed at a 95\% confidence interval (CI) for the sex of hospitalized patients with community- or nosocomial-acquired group B streptococci pneumonia. A calculation by chi-square test for two independent, ordinary regular samples of three possibilities was conducted to detect the following: whether GBS was sensitive, intermediate, or resistant to antibiotics; the differences in antibiotic resistance between the sexes; and the differences in acquisition of pneumonia between the sexes [12]. A calculation by the chi-square test for sex differences of two possibilities was performed to compare the different detection techniques of GBS and the number of deaths between the sexes. One-way analysis of variance (ANOVA) for the independent sexes was used to compare the duration of hospital stays. In addition, statistical two-sided examinations were completed. A $P$ value of $<0.05$ was considered statistically significant.

\section{Results}

Overall, 66 inpatients among 6,932 pneumonia inpatients $(1.0 \%, 95 \%$ CI $0.8 \%-1.2 \%)$, with an average age of $63.3 \pm 16.1$ years (45 males [68.2\%, 95\% CI
$60.0 \%-79.4 \%$ ] and 21 females [31.8\%, 95\% CI $20.6 \%-43.0 \%]$ ), were discovered to have communityor nosocomial-acquired pneumonia due to GBS (ICD J15.3) in the hospital records of the Department of Internal Medicine of HELIOS Clinic Wuppertal from January 1, 2004, to August 12, 2014. Males were more likely to develop pneumonia caused by GBS; however, there was no tangible inter-gender difference in antibiotic resistance (Table 1). There were 48 patients $(72.7 \%)$ with community-acquired pneumonia, 11 patients $(16.7 \%)$ with nosocomial-acquired pneumonia, and 7 patients $(10.6 \%)$ with aspiration pneumonia. A gender difference was not detected in type of acquisition of pneumonia (Table 2).

Fifty-one inpatients were eliminated from this study because either they had another infectious disorder due to GBS or because direct access to their data at the Division of Neurology was not allowed.

In this trial, the most commonly administered antibiotics for patients with pneumonia due to GBS were the combinations of piperacillin-tazobactam followed by ampicillin-sulbactam (Table 1).

Compared to gentamicin, no resistance was found to penicillin, ampicillin, piperacillin, ampicillin-sulbactam, piperacillin-tazobactam, cefuroxime, cefotaxime, or vancomycin in any inpatients with community- or nosocomial-acquired pneumonia triggered by GBS; this finding is statistically significant $(P<0.0001$; Table 1$)$. GBS had the highest level of antimicrobial resistance to gentamicin when compared to penicillin $(P<0.0001$; Table 1$)$.

GBS also had an elevated level of resistance to erythromycin when compared with gentamicin $(\boldsymbol{P}<$ 0.0001; Table 1). A statistical comparison of erythromycin, to which GBS had a high level of antimicrobial resistance, and penicillin, to which GBS had no antibiotic resistance, was also completed $(P=0.008$; Table $1)$.

GBS was most often detected in venous blood cultures in female pneumonia patients, and in tracheal secretions in male pneumonia patients (Table 2). All discovered GBS cases were from isolates of facultative pathogenic GBS strains in patients with pneumonia.

The mean duration of the hospital stay of inpatients suffering from group B streptococcal pneumonia was $14.6 \pm 14.8$ days. A gender difference in the length of hospital stay was not detected (Table 2). There were 7 actual deaths $(10.6 \%$, 95\% CI $3.2 \%-18.0 \%)$ related to group B streptococci pneumonia. Therefore, the survival rate was $89.4 \%$ (95\% CI $81.5 \%-97.3 \%)$ for all inpatients with community- or nosocomial-acquired pneumonia caused by GBS in this investigation. Although more male than female pneumonia patients died, the difference was not statistically significant (Table 2 ). 
Table 1. Resistance to various antibiotic groups after susceptibility testing, with the number of tested antibiotics and number of antibiotics used in treatment of group B streptococcal pneumonia patients and a comparison between the sexes.

\begin{tabular}{|c|c|c|c|c|c|c|c|c|c|}
\hline & & & & $\begin{array}{l}\text { No. of inpatients } \\
\text { with group B } \\
\text { Streptococcus }=66\end{array}$ & $\begin{array}{l}\text { No. of males }=45 \\
\text { No. of } \\
\text { females }=21\end{array}$ & & & & \\
\hline Drug groups & $\begin{array}{l}\text { Active } \\
\text { substance }\end{array}$ & $\begin{array}{l}\text { No. of } \\
\text { patients } \\
\text { using anti- } \\
\text { biotic }\end{array}$ & $\begin{array}{l}\text { No. of anti- } \\
\text { biotics } \\
\text { tested on } \\
\text { anti-biogram }\end{array}$ & Sensitive (\%) & $\begin{array}{l}\text { Intermediate } \\
(\%)\end{array}$ & Resistant (\%) & $\begin{array}{l}P \text { value com- } \\
\text { pared with } \\
\text { gentamicin }\end{array}$ & $\begin{array}{l}P \text { value com- } \\
\text { pared with } \\
\text { penicillin }\end{array}$ & $\begin{array}{l}P \text { value } \\
\text { male com- } \\
\text { pared with } \\
\text { female }\end{array}$ \\
\hline \multirow[t]{9}{*}{ Penicillins } & Penicillin & 1 & 66 & $66(100)$ & 0 & 0 & $<0.0001$ & & \\
\hline & Male & 0 & 45 & 45 (100) & 0 & 0 & & & 1.0 \\
\hline & Female & 1 & 21 & $21(100)$ & 0 & 0 & & & \\
\hline & Ampicillin & 2 & 66 & $66(100)$ & 0 & 0 & $<0.0001$ & 1.0 & \\
\hline & Male & 2 & 45 & $45(100)$ & 0 & 0 & & & 1.0 \\
\hline & Female & 0 & 21 & $21(100)$ & 0 & 0 & & & \\
\hline & Piperacillin & 0 & 65 & 65 (98.5) & 0 & 0 & $<0.0001$ & 1.0 & \\
\hline & Male & 0 & 45 & $45(100)$ & 0 & 0 & & & 1.0 \\
\hline & Female & 0 & 20 & $20(100)$ & 0 & 0 & & & \\
\hline \multirow{6}{*}{$\begin{array}{l}\text { Penicillin }+ \\
\text { Beta lactamase } \\
\text { inhibitors }\end{array}$} & $\begin{array}{l}\text { Ampicillin + } \\
\text { Sulbactam }\end{array}$ & 20 & 66 & $66(100)$ & 0 & 0 & $<0.0001$ & 1.0 & \\
\hline & Male & 11 & 45 & $45(100)$ & 0 & 0 & & & 1.0 \\
\hline & Female & 9 & 21 & $21(100)$ & 0 & 0 & & & \\
\hline & $\begin{array}{l}\text { Piperacillin + } \\
\text { Tazobactam }\end{array}$ & 30 & 66 & $66(100)$ & 0 & 0 & $<0.0001$ & 1.0 & \\
\hline & Male & 23 & 45 & $45(100)$ & 0 & 0 & & & 1.0 \\
\hline & Female & 7 & 21 & $21(100)$ & 0 & 0 & & & \\
\hline \multirow[t]{6}{*}{ Cephalo-sporins } & Cefuroxime & 0 & 66 & $66(100)$ & 0 & 0 & $<0.0001$ & 1.0 & \\
\hline & Male & 5 & 45 & $45(100)$ & 0 & 0 & & & 1.0 \\
\hline & Female & 3 & 20 & $20(100)$ & 0 & 0 & & & \\
\hline & Cefotaxime & 0 & 66 & $66(100)$ & 0 & 0 & $<0.0001$ & 1.0 & \\
\hline & Male & 0 & 45 & $45(100)$ & 0 & 0 & & & 1.0 \\
\hline & Female & 0 & 21 & $21(100)$ & 0 & 0 & & & \\
\hline \multirow[t]{6}{*}{ Gyrase inhibitors } & Ciprofloxacin & 2 & 30 & 0 & $29(96.7)$ & $1(3.3)$ & 0.393 & $<0.0001$ & \\
\hline & Male & 2 & 21 & $18(85.7)$ & $1(4.8)$ & $2(9.5)$ & & & 0.741 \\
\hline & Female & 0 & 11 & 0 & $11(100)$ & 0 & & & \\
\hline & Levofloxacin & 0 & 54 & 17 (31.5) & $36(66.6)$ & $1(1.9)$ & $<0.0001$ & $<0.0001$ & \\
\hline & Male & 2 & 36 & $9(25.0)$ & $26(72.2)$ & $1(2.8)$ & & & 0.295 \\
\hline & Female & 0 & 18 & $8(44.4)$ & 10 (55.6) & 0 & & & \\
\hline \multirow[t]{3}{*}{ Macrolides } & Erythromycin & 1 & 66 & $57(86.4)$ & $1(1.5)$ & $8(12.1)$ & $<0.0001$ & 0.008 & \\
\hline & Male & 0 & 45 & $40(88.9)$ & 0 & $5(11.1)$ & & & 0.306 \\
\hline & Female & 1 & 21 & 17 (80.9) & $1(4.8)$ & $3(14.3)$ & & & \\
\hline \multirow{3}{*}{$\begin{array}{l}\text { Trimethoprim + } \\
\text { Sulfonamide }\end{array}$} & Co-trimoxazole & 0 & 15 & 13 (86.7) & 0 & $2(13.3)$ & $<0.0001$ & 0.011 & \\
\hline & Male & 0 & 11 & $10(90.9)$ & 0 & $1(9.1)$ & & & 0.726 \\
\hline & Female & 0 & 4 & $3(75.0)$ & 0 & $1(25.0)$ & & & \\
\hline \multirow[t]{3}{*}{ Lincosamides } & Clindamycin & 2 & 66 & $59(89.4)$ & $1(1.5)$ & $6(9.1)$ & $<0.0001$ & 0.025 & \\
\hline & Male & 2 & 45 & 41 (91.1) & $1(2.2)$ & $3(6.7)$ & & & 0.489 \\
\hline & Female & 0 & 21 & 18 (85.7) & 0 & $3(14.3)$ & & & \\
\hline \multirow[t]{3}{*}{ Amino-glycosides } & Gentamicin & 0 & 66 & 0 & $58(87.9)$ & $8(12.1)$ & & $<0.0001$ & \\
\hline & Male & 0 & 45 & 0 & $40(88.9)$ & $5(11.1)$ & & & 0.932 \\
\hline & Female & 0 & 21 & 0 & $18(85.7)$ & $3(14.3)$ & & & \\
\hline \multirow[t]{3}{*}{ Glyco-peptides } & Vancomycin & 0 & 66 & $66(100)$ & 0 & 0 & $<0.0001$ & 1.0 & \\
\hline & Male & 0 & 45 & $45(100)$ & 0 & 0 & & & 1.0 \\
\hline & Female & 0 & 21 & $21(100)$ & 0 & 0 & & & \\
\hline
\end{tabular}

Note: All significant $P$ values are shown in bold.

\section{Discussion}

During the 10-year period of this qualitative, observational control study, GBS did not develop resistance to the following antibiotics used in the therapy of inpatients treated for diagnosed community- or nosocomial-acquired pneumonia: penicillin, ampicillin, piperacillin, ampicillin-sulbactam, piperacillin-tazobactam, cefuroxime, cefotaxime, and vancomycin. So-called community-acquired pneumonia is a typical acute lower-airway disease that is most com- monly triggered by GBS and is one of the principal causes of increasing numbers of deaths around the world. This study found that GBS pneumonia constitutes only about $1 \%$ of the thousands of pneumonia cases in the period investigated. That undermines, to an extent, a common impression that these bacteria are some of the most frequent causative pneumonia-prone pathogens, particularly in the elderly [13]. Pneumonia is a manifestation of GBS disease that occurs almost exclusively in older debilitated adults. The association of pneumonia with central nervous 
system dysfunction from dementia, neurological impairment from cardiovascular disease, or encephalopathy suggests that aspiration is an important antecedent to the development of lower respiratory involvement [14].

Table 2. The different identification procedures and acquisition of group B Streptococcus, duration of hospital stay, and number of deaths in hospitalized patients with community- or nosocomial-acquired pneumonia, compared between sexes.

\begin{tabular}{|c|c|c|c|}
\hline Samples & $\begin{array}{l}\text { Male } \\
(\mathrm{n}=45)(\%)\end{array}$ & $\begin{array}{l}\text { Female } \\
(\mathrm{n}=21)(\%)\end{array}$ & $P$ value \\
\hline Bronchial secretion & $11(24.4)$ & $5(23.8)$ & 0.807 \\
\hline Tracheal secretion & $16(35.6)$ & $3(14.3)$ & 0.137 \\
\hline Sputum & $5(11.1)$ & $3(14.3)$ & 1.0 \\
\hline Throat swab & $1(2.2)$ & 0 & 0.699 \\
\hline Venous blood culture & $12(26.7)$ & $9(42.9)$ & 0.303 \\
\hline Secretion drainage & 0 & $1(4.8)$ & 0.699 \\
\hline Acquisition of pneumonia & & & 0.266 \\
\hline $\begin{array}{l}\text { Community-acquired } \\
\text { pneumonia }\end{array}$ & $30(66.7)$ & $18(85.7)$ & \\
\hline $\begin{array}{l}\text { Nosocomial-acquired } \\
\text { pneumonia }\end{array}$ & $9(20.0)$ & $2(9.5)$ & \\
\hline Aspiration pneumonia & $6(13.3)$ & $1(4.8)$ & \\
\hline $\begin{array}{l}\text { Duration of hospital stay, } \\
\text { mean + SD (days) }\end{array}$ & $15.8 \pm 16.6$ & $11.3 \pm 10.5$ & 0.264 \\
\hline No. of deaths & $5(11.1)$ & $2(9.5)$ & 0.823 \\
\hline
\end{tabular}

SD: standard deviation

The first antibacterial therapy is usually empiric for patients with pneumonia caused by GBS. For these patients, the results of bacteriological cultivation and antimicrobial susceptibility testing are seldom obtainable when the pneumonia due to GBS is first diagnosed. Any antibiotic selected for empiric therapy should be an effective treatment against GBS, have a good drug tolerance, and be able to be given in single doses. Group B streptococci are the primary cause of pneumonia, despite the differing frequencies of the appearance of this feared bacterium worldwide. Substantial geographic differences exist around the world in the level of group B streptococci resistance to penicillin $[15,16]$.

In the long run, antibiotics such as penicillin lose their effect on group B streptococci. The consequence of this is that the frequency of group B streptococcal pneumonia has progressively increased during the last three decades all over the world [17]. One possible explanation for penicillin resistance is that the overuse of antibiotics for the treatment of pneumonia and the abuse of antibiotics for viral infections of the upper airways have resulted in increased antimicrobial resistance of GBS to penicillin [18]. In contrast to previous reports, no resistance to penicillin was found in the isolates from respiratory specimens in the present study over the last 10 years. An explanation for this cannot be provided through this study. There are apparently still significant geographical differences in the levels of the antimicrobial resistance of GBS to penicillin. Therefore, all laboratories should be encouraged to record the sensitivities of several antibiotics used in their territories simultaneously, in order to allow clinicians to advise the use of the most appropriate antibiotic agents in the initial treatment of group B streptococcal pneumonia. This study showed that penicillin is still a suitable antibiotic for the successful management of group B streptococcal pneumonia.

A previous study identified a decreased susceptibility to ampicillin in pneumococcal isolates discovered in sputum [19]. In the case of severe pneumonia, this earlier study suggested a combination of amoxicillin therapy with a macrolide or fluoroquinolone because of the increasing development of resistance in pneumococcal isolates to amoxicillin [19]. Despite the small administration rate of ampicillin as a therapeutic agent in this study, it can be administered alone, according to the susceptibility testing, for the treatment of pneumonia, particularly in the worst-case scenario of the discovery of group B streptococci in sputum. In this study, GBS was not resistant to ampicillin in patients with pneumonia in the last decade.

Microbiological tests also detected no antibiotic resistance in group B streptococci to piperacillin in this study. Piperacillin has extensive effectiveness on Gram-positive and Gram-negative microorganisms; moreover, it has shown higher activity against beta-lactamase-producing bacteria than other penicillins. In an earlier study, the activity of piperacillin was examined in GBS isolated from patients with infectious diseases of the respiratory tract. From these results, piperacillin was detected to still be highly effective currently in relation to the maintenance of favorable antibacterial activities in several bacterial infections [20].

In another previous study, the efficacy of ampicillin-sulbactam in the antibiotic management of inpatients suffering from pneumococcal pneumonia was examined [21]. Ampicillin-sulbactam is a combination beta-lactamase inhibitor antibiotic that is commonly used in hospitals against a large variety of microorganisms on the Gram-positive and Gram-negative spectrum. The rate of antibiotic resistance to ampicillin was $43 \%$ in that previous study, and it was concluded that ampicillin-sulbactam showed excellent general clinical effectiveness in the antibiotic medication of inpatients with community-acquired pneumonia [21]. In the present study, GBS did not develop resistance to the ampicillin-sulbactam combination, which was frequently used as an antibiotic medication for the treatment of 
patients diagnosed with community- and nosocomial-acquired pneumonia.

Another beta-lactam-inhibitor combination is piperacillin-tazobactam, with wide-ranging antibacterial effectiveness on Gram-positive and Gram-negative bacteria. Piperacillin is administered together with the beta-lactamase inhibitor tazobactam exclusively to cancel resistance of bacteria to the antibiotic. The effectiveness of piperacillin-tazobactam has been proven in previous studies [22]. While still showing excellent activity, resistance to piperacillin-tazobactam has increased slightly in recent years, according to reports [22]. This study could not demonstrate the development of resistance of GBS to piperacillin-tazobactam, which increases the usefulness of the piperacillin-tazobactam combination for the treatment of patients with group B streptococci pneumonia.

Cefuroxime produced great susceptibility testing results in hospitalized patients who were suffering from streptococcal pneumonia in this study, and it is recommended for the medication of pneumonia patients. All test samples of GBS were sensitive to cefuroxime in this study. Clinical investigations have reported that cefuroxime is an effective antibiotic drug for acute infectious diseases of the respiratory tract and other infections [23].

Second-generation cephalosporins are also advised for the first-line treatment of pneumonia. Nevertheless, reports of cefuroxime therapy for penicillin-resistant pneumococcal pneumonia are very limited, and they do not include cefuroxime-resistant cases [24].

A third-generation cephalosporin, cefotaxime, exhibited excellent activity in the susceptibility testing in the present investigation. Due to its broad-spectrum activity, cefotaxime is used for the treatment of serious infectious diseases, mainly severe pneumonia, in clinical practice [25].

Although cefuroxime and cefotaxime showed effective activity on GBS in the susceptibility testing, neither of these two cephalosporins was used in the treatment of streptococcal pneumonia patients in this study. The non-use of cefotaxime could possibly be attributed to an increase in resistance to penicillin and cefotaxime after 1996 [26].

Susceptibility tests in this investigation revealed that GBS strains were extremely sensitive to vancomycin, an antibiotic of the glycopeptide class, which is used in the treatment of infectious diseases caused by Gram-positive bacteria [27]. Despite increasing antibiotic resistance in recent years, vancomycin is the only antimicrobial agent to which group B streptococci, the most important cause of bacterial pneumonia, has not become resistant [27].
The fluoroquinolones contain a family of associated chemical composites, such as ciprofloxacin, levofloxacin, etc. The prescription of this medication for the management of pneumonia represents major therapeutic progress. At the time of their implementation, these broad-spectrum antibiotics were also equally effective against GBS. Despite a very small resistance rate of GBS to ciprofloxacin and levofloxacin, as was found in the present study, fluoroquinolones continue to be used in the present day for the antimicrobial medical treatment of diverse microorganisms in the Gram-positive and Gram-negative range [28].

The development of resistance of GBS to co-trimoxazole has been increasingly reported throughout the world [29]. Co-trimoxazole is a combination of two antimicrobial agents, trimethoprim and sulfamethoxazole, developed in order to improve their positive synergistic effectiveness against Gram-positive and Gram-negative bacteria [29]. However, an increasing rate of group B streptococci resistance to co-trimoxazole was observed over the past 10 years in this study. Co-trimoxazole is not commonly prescribed for the treatment of pneumonia in industrialized countries, but it is often used for the treatment of opportunistic infections, including pneumonia, in developing countries [29].

Macrolides or lincosamides should not be used empirically for severe group B streptococcal pneumonia before streptococcal strains are found to be sensitive in susceptibility testing [30]. A high resistance rate to erythromycin and clindamycin was also found in the current study.

Although penicillin remains the first antibiotic of choice for treating pneumonia, macrolides such as erythromycin have been used for patients with allergies to beta-lactams and in combination therapies for severe pneumonia. While other studies have recommended a combination therapy with penicillin and gentamicin against penicillin-resistant GBS, the current investigation contradicts these studies with the confirmation of a high incidence of gentamicin resistance in GBS. Gentamicin was used most frequently for the antimicrobial treatment of pneumonia in a previous study [31].

\section{Study Limitations}

The results of this study describe the long-term evaluation of antimicrobial resistance in GBS at a single large university hospital; thus, the outcomes of this study cannot easily be applied to other geographic regions. Additionally, after an evaluation of this study, it became apparent that not all of the antibiotics were tested at the same frequency when pre- 
paring the antibiograms of inpatients with group B streptococcal pneumonia.

\section{Conclusions}

All of the inpatients with group B streptococcal pneumonia in this study exhibited antibiotic resistance to gyrase inhibitors, macrolides, co-trimoxazole, lincosamides, and aminoglycosides. None of these same patients exhibited antibiotic resistance to penicillin, cefuroxime, cefotaxime, or vancomycin.

\section{Competing Interests}

The authors have declared that no competing interest exists.

\section{References}

1. Krzyściak W, Pluskwa KK, Jurczak A, Kościelniak D. The pathogenicity of the Streptococcus genus. Eur J Clin Microbiol Infect Dis. 2013;32:1361-76.

2. LaRock CN, Nizet V. Cationic antimicrobial peptide resistance mechanisms of streptococcal pathogens. Biochim Biophys Acta. 2015; S0005-2736(15)00050-4.

3. Persson E, Berg S, Bergseng H, Bergh K, Valsö-Lyng R, Trollfors B. Antimicrobial susceptibility of invasive group B streptococcal isolates from south-west Sweden 1988-2001. Scand J Infect Dis. 2008;40:308-13.

4. World Health Organization (WHO). International Classification of Diseases (ICD). http://www who.int/classification/icd/en/. Accessed March 9, 2015.

5. Bodmann KF. Current guidelines for the treatment of severe pneumonia and sepsis. Chemotherapy. 2005;51:227-33.

6. Niederman MS, Mandell LA, Anzeuto A, et al. Guidelines for the management of adults with community-acquired pneumonia. Diagnosis, assessment of severity, antimicrobial therapy, and prevention. Am J Respir Crit Care Med. 2001;163:1730-54.

7. Watkins RR, Lemonovich TL. Diagnosis and management of community-acquired pneumonia in adults. Am Fam Physician. 2011;83:1299-1306.

8. Clinical and Laboratory Standards Institute. Performance standards for antimicrobial susceptibility testing. CLSI M100-S22. Wayne (PA): Clinical and Laboratory Standards Institute. 2012.

9. European Committee on Antimicrobial Susceptibility Testing (EUCAST) breakpoints 2011-2014. http:/ / www.eucast.org. Accessed March 9, 2015.

10. Bauer AW, Kirby WM, Sherris JC, Turck M. Antibiotic susceptibility testing by a standardized single disk method. Am J Clin Pathol. 1966;45:493-6.

11. Barlett JG. Diagnosis of bacterial infections of the lung. Clin Chest Med. 1987:8:119-34

12. VassarStats [website for statistical computation] and Concepts \& applications of inferential statistics [companion textbook]. http://vassarstats.net/ and http://vassarstats.net/textbook/. Accessed March 22, 2015.

13. Edwards MS, Baker CJ. Group B streptococcal infections in elderly adults. Clin Infect Dis. 2005;41:839-47.

14. Verghese A, Berk SL, Boelen LJ, Smith JK. Group B streptococcal pneumonia in the elderly. Arch Intern Med. 1982;142:1642-5.

15. Betriu C, Gomez M, Sanchez A, Cruceyra A, Romero J, Picazo JJ. Antibiotic resistance and penicillin tolerance in clinical isolates of group B streptococci. Antimicrob Agents Chemother. 1994;38:2183-6.

16. Kasahara K, Baltus AJ, Lee SH, Edelstein MA, Edelstein PH. Prevalence of non-penicillin-susceptible group B streptococcus in Philadelphia and specificity of penicillin resistance screening methods. J Clin Microbiol. 2010;48:1468-9.

17. Kimura K, Suzuki S, Wachino J, Kurokawa H, Yamane K, Shibata N, et al. First molecular characterization of group B streptococci with reduced penicillin susceptibility. Antimicrob Agents Chemother. 2008;52:2890-7.

18. Seki T, Kimura K, Reid ME, Miyazaki A, Banno H, Jin W, et al. High isolation rate of MDR group B streptococci with reduced penicillin susceptibility in Japan. J Antimicrob Chemother. 2015;70:2725-8.

19. Bédos JP. [Would ampicillin alone again be enough for treating community-acquired pneumonias?]. Presse Med 1998; 27:1440-1441. [Article in French]

20. Holmes B, Richards DM, Brogden RN, Heel RC. Piperacillin. A review of its antibacterial activity, pharmacokinetic properties and therapeutic use. Drugs. 1984:28:375-425.

21. Williams D, Perri M, Zervos MJ. Randomized comparative trial with ampicillin/sulbactam versus cefamandole in the therapy of community acquired pneumonia. Eur J Clin Microbiol Infect Dis. 1994;13:293-8.

22. Marshall SA, Aldridge KE, Allen SD, Fuchs PC, Gerlach EH, Jones RN. Comparative antimicrobial activity of piperacillin-tazobactam tested against more than 5000 recent clinical isolates from five medical centers. A reevaluation after five years. Diagn Microbiol Infect Dis. 1995;21:153-68.
23. Smith BR, LeFrock JL. Cefuroxime: antimicrobial activity, pharmacology, and clinical efficacy. Ther Drug Monit. 1983; 5:149-60.

24. Niederman MS, Bass JB Jr, Campbell GD, et al. Guidelines for the initial management of adults with community-acquired pneumonia: diagnosis, assessment of severity, and initial antimicrobial therapy. American Thoracic Society. Medical Section of the American Lung Association. Am Rev Respir Dis. 1993;148:1418-26.

25. Robertson MB, Korman TM, Dartnell JG, et al.; Victorian Drug Usage Evaluation Group. Ceftriaxone and cefotaxime use in Victorian hospitals. Med J Aust. 2002;176:524-29

26. Brett MS, Martin DR. A significant increase in antimicrobial resistance among pneumococci causing invasive disease in New Zealand. $\mathrm{N} \mathrm{Z}$ Med J. 1999;112:113-5.

27. Stevens DL. The role of vancomycin in the treatment paradigm. Clin Infect Dis. 2006;42(Suppl 1):S51-S57.

28. Wang P, Tong JJ, Ma XH, Song FL, Fan L, Guo CM, et al. Serotypes, antibiotic susceptibilities, and multi-locus sequence type profiles of Streptococcus agalactiae isolates circulating in Beijing, China. PLoS One. 2015;10:e0120035.

29. Traub WH. Leonhard B. Comparative susceptibility of clinical group A, B, C, $\mathrm{F}$, and $\mathrm{G}$ beta-hemolytic streptococcal isolates to 24 antimicrobial drugs. Chemotherapy. 1997;43:10-20.

30. Megged O, Assous M, Weinberg G, Schlesinger Y. Inducible clindamycin resistance in $\beta$-hemolytic group B streptococci and Streptococcus pneumoniae. Isr Med Assoc J. 2013;15:27-30.

31. Dworzack DL, Hodges GR, Barnes WG, Rosett W. Group B streptococcal infections in adult males. Am J Med Sci. 1979;277:67-73. 